Pacific Journal of Mathematics

DIRICHLET'S THEOREM FOR THE RING OF POLYNOMIALS 


\section{DIRICHLET'S THEOREM FOR THE RING OF POLYNOMIALS OVER GF(2)}

\section{Douglas Hensley}

Let $G$ denote the ring $\mathrm{GF}(2)[x]$ of polynomials $g(x)$ over the field of integers mod 2 . Let

$$
I(k)=\#\{p \in G: \operatorname{deg} p=k \text { and } p \text { is irreducible in } G\} .
$$

It is well known that $I(k)=(1 / k) \sum_{d \mid k} \mu(d) 2^{k / d}$. Here we prove an analog to Dirichlet's Theorem on primes in arithmetic progressions. For any $m \in G$ the $p$ counted in $I(k)$ are uniformly distributed among the congruence classes $(b) \bmod m$ for which $(b, m)=1$. The result is especially sharp when $m$ is square-free.

1. Introduction and notation. As in the abstract, $G=\mathrm{GF}(2)[x]$. We will suppress the variable and write, for instance, 1011 in place of $x^{3}+x+1$. We denote the set of irreducible $p \in G$ by $I$. The only part of this work which does not seem to generalize easily to other $\operatorname{GF}(q)[x], q$ a prime, is the special role of square-free moduli. Defining $\phi: G \rightarrow Z$ in the natural way $(\phi(m)=\#\{a: \operatorname{deg} a=\operatorname{deg} m$ and $(a, m)=1\})$, we have that

$\phi(m)$ is odd if and only if $m$ is square-free.

Consequently, none of the "Dirichlet characters" on $G / m G$ can have as their range $\{-1,0,1\}$. The absence of this kind of Dirichlet character permits sharper bounds. For fixed $m \in G, b \in G$ with $(b, g)=1$, let $I_{b}(n)$ denote the number of irreducible $p \in G$ of degree $n$ such that $p \equiv b \bmod m$.

THEOREM. There exist positive effectively computable constants $C_{1}$ and $C_{2}$ such that for all integers $M, N \geq 1$, for all square-free polynomials $m \in G$ of degree $M$, and for all congruence classes $(b) \bmod m$ relatively prime to $m$,

$$
\left|I_{b}(N)-\frac{2^{N}}{N \phi(m)}\right| \leq \frac{C_{1} M 2^{N}}{N} \exp \left(-C_{2} N M^{-9}(\log M)^{-3}\right) .
$$


That is,

$$
I_{b}(N)=\frac{2^{N}}{N \phi(m)}\left(1+O\left(M \phi(m) e^{-C_{2} N M^{-9}(\log M)^{-3}}\right)\right)
$$

uniformly in $N, M, m$ and $b$.

The result, of course does not constitute any improvement on the trivial bounds $0 \leq I_{b}(N) \leq I(N)$ unless $N$ is larger, roughly, than $M^{9}$. It differs from results of Uchiyama and Carlitz $[1,3,4]$ in its generality and uniformity with respect to the modulus, treating the ring $G$ as fixed. Basically they kept $G$ variable and constrained $m$.

When $m$ is not square-free, characters of the second kind intrude, and we must settle for $2^{-M} M^{-2}$ in place of $M^{-9}(\log M)^{-3}$ in Theorem 1 .

2. Preliminaries. For much of its length our proof follows the path of the classic proof of Dirichlet's theorem. There are analogs to Dirichlet characters, to $L$-functions, and product expansions valid in a half-plane. The difference is that in this case the $L$-functions are essentially polynomial functions on $\mathbf{C}$. This simplifies the analysis. We can dispense with contour integrations, and just compare coefficients in two expansions of

$$
\sum_{\chi \bmod m} \frac{1}{\chi(b)} \frac{L^{\prime}(s, \chi)}{L(s, \chi)},
$$

as series in $t=2^{-s}$. The reader who wants to see just what is different can skip this section.

Let $N a=2^{\operatorname{deg} a}$, for $a \in G$. Let

$$
\phi(m)=\#\{a: \operatorname{deg} a=\operatorname{deg} m \text { and }(a, m)=1\} .
$$

Note that for $p \in G$ irreducible, $\phi(p)=N p-1$ and is odd. Finally, the usual proof that

$$
\phi(m)=(N m) \prod_{p \mid m}\left(1-\frac{1}{N p}\right)
$$

is valid in this setting too, so $\phi(m)$ is multiplicative. Thus $\phi(m)$ is odd if and only if $m$ is square-free.

A character $\bmod m$ is a function $\chi: G \rightarrow \mathbf{C}$ such that

$$
\begin{array}{lll}
\text { (i) } & \chi(a) \chi(b)=\chi(a b) & \text { for } a, b \in G . \\
\text { (ii) } & \chi(a)=\chi(b) & \text { if } a \equiv b \bmod m \\
\text { (iii) } & \chi(a)=0 & \text { for }(a, m) \neq 1 .
\end{array}
$$


As with characters in the integers, $\chi(1)=1$, and if $(a, m)=1$ then $\chi(a)$ is a $\phi(m)$ th root of 1 . For every $m$ except $1,10,11$ and 110 , there is a character other than the trivial character $\chi_{0}$, where

$$
\chi_{0}(a)=1 \quad \text { for }(a, m)=1, \quad \chi_{0}(a)=0 \quad \text { otherwise. }
$$

Further, with the same exceptions,

$$
\begin{gathered}
\sum_{a \bmod m} \chi(a)=0 \text { for all } \chi \neq \chi_{0} \\
\sum_{\chi \bmod m} \chi(a)=0 \quad \text { for all } a \neq 1 \bmod m .
\end{gathered}
$$

(All irreducibles except the factors of $m$ are $\equiv 1 \bmod m$ when $m=1,10$, 11 or 110 , since only $1 \bmod m$ is relatively prime to $m$ in these cases. From now on, we assume $m$ is not $1,10,11$ or 110.)

$$
\sum_{\chi \bmod m} \chi(1)=\phi(m)
$$

and

$$
\sum_{a \bmod m} \chi_{0}(a)=\phi(m)
$$

Proof. The classical proofs go over word for word. See e.g. Landau [2].

We now define a power series $f_{\chi}(t)$ corresponding to each $\chi \bmod m$. With the substitution $t=2^{-s}$ we get the analog of a Dirichlet $L$-series.

\section{DEFINITION.}

$$
f_{\chi}(t)=\sum_{a \in G} \chi(a) t^{\operatorname{deg} a}=\sum_{j=0}^{\infty}\left\{\sum_{\operatorname{deg} a=j} \chi(a)\right\} t^{j},
$$

and

$$
L(s, \chi)=\sum_{\substack{a \in G \\ a \neq 0}} \chi(a)(N a)^{-s} .
$$

Let $C_{j}(\chi)=\sum_{\operatorname{deg} a=j} \chi(a)$. Then by (2.4), for $\chi=\chi_{0}, C_{j}(\chi)=0$ for $j \geq \operatorname{deg} m$.

Thus for $\chi \neq \chi_{0}$, and with $M=\operatorname{deg} m$,

$$
f_{\chi}(t)=\sum_{j=0}^{m-1} C_{J}(\chi) t^{j}
$$

and is a polynomial over the complex numbers of degree $\leq M-1$. We note here that

$$
f_{\chi}(0)=1, \quad f_{\chi}(1)=0, \text { and }\left|C_{j}\right| \leq 2^{j}
$$


If we forget temporarily that $f_{\chi}(t)$ is a polynomial, it is natural to ask for a product expansion. Formally,

$$
f_{\chi}(t)=\prod_{p \in I}\left(1-\frac{\chi(p)}{(N p)^{s}}\right)^{-1}=\prod_{p \in I}\left(1-\chi(p) t^{\operatorname{deg} p}\right)^{-1},
$$

and the product converges absolutely for $|t|<\frac{1}{2}(\operatorname{Re}(s)>1)$. The function corresponding to the Riemann zeta function here is

$$
Z(t):=\sum_{a \neq 0} t^{\operatorname{deg} a}=\frac{1}{1-2 t},
$$

and this has the product expansion

$$
Z(t)=\prod_{k=1}^{\infty}\left(1-t^{k}\right)^{-I(k)}
$$

Finally, for $\chi=\chi_{0} \bmod m$,

$$
f_{\chi_{0}}(t)=Z(t) \prod_{p \mid m}\left(1-t^{\operatorname{deg} p}\right) .
$$

The well known identity

$$
I(k)=\frac{1}{k} \sum_{d \mid k} \mu(d) 2^{k / d}
$$

now follows from a (much) simplified reprise of the proof of the prime number theorem. We have $Z^{\prime}(t) / Z(t)=2 /(1-2 t)$ on one hand, while from (2.13) it is $\sum_{k=1}^{\infty} k I(k) t^{k-1} /\left(1-t^{k}\right)$. Expanding both sides as series about $t=0$ and equating coefficients gives

$$
2^{k}=\sum_{d \mid k} d I(d)
$$

which is equivalent to (2.15).

The same ideas feature in the proof of Theorem 1: differentiate $\log f_{\chi}(t)$, use the product formula on one side, expand things as series in $t$ and equate coefficients.

3. Partial fractions. For $\chi=\chi_{0} \bmod m$,

$$
f_{\chi_{0}}(t)=\frac{1}{1-2 t} \prod_{p \mid m}\left(1-t^{\operatorname{deg} p}\right)
$$

for $t \neq 1 / 2$. With the notations $I_{m}(k)=\#\{p \in I: p \mid m$ and $\operatorname{deg} p=k\}$, $e(r)=e^{2 \pi i r}$, we have

$$
\frac{f_{\chi_{0}}^{\prime}(t)}{f_{\chi_{0}}(t)}=\frac{2}{1-2 t} \sum_{k=1}^{M} \frac{1}{k} I_{m}(k) \sum_{j=0}^{k-1} \frac{1}{t-e(j / k)}
$$


which has simple poles at $t=1 / 2$ and at various roots of unity. In all, there are $1+\sum_{k=1}^{M} k I_{m}(k)$ poles of $\left(f_{\chi_{0}}^{\prime} / f_{\chi_{0}}\right)(t)$, and for $m$ square-free, this is just $M+1$. Now for any polynomial $f(t)$ over $\mathbf{C}$ with zeros $w_{1}$, $w_{2}, \ldots, w_{j}$ to multiplicity $N_{1}, N_{2}, \ldots, N_{j}$,

$$
\frac{f^{\prime}(t)}{f(t)}=\sum_{i=1}^{j} \frac{N_{i}}{t-w_{l}} \text {. }
$$

Thus for any character $\chi \neq \chi_{0} \bmod m$,

$$
\frac{f_{\chi}^{\prime}(t)}{f_{\chi}(t)}=\sum_{w \in \Omega_{\chi}} \frac{N(w)}{t-w},
$$

where $\Omega_{\chi}$ is the set of zeros of $f_{\chi}(t)$ and $N(w)$ the corresponding multiplicity, for $w \in \Omega_{\chi}$. By (2.11), $f_{\chi}(t) \neq 0$ for $|t|<1 / 2$, that is, $|w| \geq 1 / 2$ if $w \in \Omega_{\chi}$. We now fix $b \bmod m,(b, m)=1$, and consider

$$
\sum_{\chi \bmod m} \frac{1}{\chi(b)} \frac{f_{\chi}^{\prime}(t)}{f_{\chi}(t)} \text {. }
$$

On one hand, this is equal to

$$
\begin{gathered}
\sum_{\substack{\chi \bmod _{\chi} \\
\chi \neq \chi_{0}}} \frac{1}{\chi(b)} \sum_{w \in W_{\chi}} \frac{N(w)}{t-w}+\frac{2}{1-2 t} \\
-\sum_{k=1}^{M} \frac{1}{k} I_{m}(k) \sum_{j=0}^{k-1} \frac{1}{t-e(j / k)} .
\end{gathered}
$$

We anticipate that for small $t$, the series expansion of this about zero converges, and that the dominant contribution to the coefficient of $t^{n}$ for large $n$ comes from $2 /(1-2 t)$.

On the other hand, (3.4) equals

$$
\begin{aligned}
\sum_{\chi \bmod m} \frac{1}{\chi(b)} & \sum_{p \in I} \frac{\chi(p)(\operatorname{deg} p) t^{\operatorname{deg} p-1}}{1-\chi(p) t^{\operatorname{deg} p}} \\
& =\sum_{k=1}^{\infty} k \sum_{j=0}^{\infty} \sum_{p \in I} \sum_{\chi \bmod m} \frac{1}{\chi(b)}(\chi(p))^{J+1} t^{(J+1) k-1} \\
& =\sum_{n=1}^{\infty} n t^{n-1} \sum_{d \mid n} \frac{1}{d} \sum_{\substack{p \in I \\
\operatorname{deg} p=n / d}} \sum_{\bmod m} \frac{1}{\chi(b)}(\chi(p))^{d} .
\end{aligned}
$$

Thus the coefficient of $t^{n-1}$ in the expansion of (3.6) about $t=0$ is

$$
n \sum_{d \mid n} \frac{1}{d} \sum_{\substack{p \in I \\ \operatorname{deg} p=n / d}} \sum_{\chi \bmod m} \frac{1}{\chi(b)} \chi(p)^{d} .
$$


In (3.7), the part due to $d=1$ is predominant, as we shall see. This part simplifies by (2.5) and (2.6) to

$$
n \phi(m) \sum_{\substack{p \in I \\ \operatorname{deg} p=n}} 1=n \phi(m) I_{b}(n) .
$$

The other terms may be estimated rather crudely. For any $d$,

$$
\left|\sum_{\chi \bmod m} \frac{1}{\chi(b)} \chi(p)^{d}\right| \leq \varphi(m),
$$

and $I(d) \leq 2^{d} / d$. Thus in (3.7) the part of the sum due to a particular $d$ has absolute value $\leq(n / d) 2^{d} \phi(m)$.

This gives

$$
\sum_{\chi \bmod m} \frac{1}{\chi(b)} \frac{f_{\chi}^{\prime}(t)}{f_{\chi}(t)}=\sum_{n=1}^{\infty} n \phi(m)\left\{I_{b}(n)+O\left(\frac{1}{n} 2^{n / 2}\right)\right\} t^{n-1}
$$

The implicit constant is independent of $b, m$, and $n$.

In (3.5) the expansion of $2 /(1-2 t)$ is simple, and the coefficients of $t^{n}$ arising from $1 /(t-e(j / k))$ are quite small by comparison. We just need a bound on $|w|$ for $w \in \Omega_{\chi}, \chi \neq \chi_{0}$. Here the distinction between characters of the second kind (real valued and taking -1 as well as +1 ) and third kind (not real) is important.

If $\chi$ is a character of the second kind then following Landau's treatment in [2] one sees that $f_{\chi}(1 / 2) \neq 0$. But then

$$
f_{\chi}(1 / 2)=\sum_{j=0}^{M-1} C_{j}(1 / 2)^{j}
$$

and $c_{J}=\sum_{\operatorname{deg} a=j} \chi(a)$ is an integer here, so $\left|f_{\chi}(1 / 2)\right| \geq 2^{-M}$. More sophisticated approaches led to no better an estimate. The estimate for $I_{b}(n)$ when $m$ is not square-free is done the same way as that for when $m$ is square-free, except at this point. Since the main interest attaches to the uniformly good estimates to be had for square-free $m$, we shall not go into this any more.

Assume now that $m$ is square-free. Then there are no real characters other than $\chi_{0}$.

4. The zeros of $f_{\chi}(t)$ for characters of the third kind. By the familiar device based on the inequality $3+4 \cos \theta+\cos 2 \theta \geq 0$ and the product expansion (2.11), we have

$$
\left|f_{\chi_{0}}^{3}(t) f_{\chi}^{4}(t) f_{\chi^{2}}(t)\right| \geq 1 \text { for }|t|<1 / 2 .
$$


Since $\chi$ takes on non-real values, $\chi^{2} \neq \chi_{0}$, so $\left|f_{\chi^{2}}(t)\right| \leq M$ for $|t| \leq 1 / 2$. The factor involving $\chi_{0}$ is easily estimated:

$$
\left|f_{\chi_{0}}(t)\right| \leq\left|\frac{1}{1-2 t}\right| \prod_{p \mid m}\left(1+\frac{1}{N p}\right), \text { for }|t|<\frac{1}{2} .
$$

It is well known that for integer $n \rightarrow \infty, \phi(n) \gg n / \log \log n$; the worst case is when $n$ is the product of the first $k$ primes for some $k$.

Similarly here we have for $\operatorname{deg} m=M, M \rightarrow \infty$ that

$$
\phi(m) \gg 2^{M} / \log M, \text { uniformly in } m \text {. }
$$

Since

$$
\begin{gathered}
\prod_{p \mid m}\left(1+\frac{1}{N p}\right)<\prod_{p \mid m}\left(1-\frac{1}{N p}\right)^{-1}=\frac{2^{M}}{\phi(m)}, \\
\prod_{p \mid m}\left(1+\frac{1}{N p}\right) \ll \log M,
\end{gathered}
$$

and so

$$
\left|f_{\chi_{0}}(t)\right| \ll\left|\frac{\log M}{1-2 t}\right|, \quad|t|<\frac{1}{2} .
$$

Now from (4.1),

(4.4) $\quad\left|f_{\chi}(t)\right| \gg M^{-1 / 4}(\log M)^{-3 / 4}|t-1 / 2|^{3 / 4}$ in $|t|<1 / 2$.

To estimate $f_{\chi}^{\prime}(t) / f_{\chi}(t)$ we also need an upper bound for $f_{\chi}^{\prime}(t)=$ $\sum_{j=1}^{m-1} j C_{j} t^{j-1}$, in $|t|<1 / 2$.

Each $\left|C_{j}\right| \leq 2^{j}$, so $\left|C_{J} t^{j-1}\right| \leq 2$. Thus

$$
\left|f_{\chi}^{\prime}(t)\right| \leq M^{2} \text { for }|t| \leq 1 / 2 \text {. }
$$

Since no polynomial can have a zero of fractional order, for fixed $\chi$, $\left|f_{\chi}(t)\right| \gg 1$ in $|t|<1 / 2$. But for variable $M$, we need a lemma.

Lemma. Uniformly in $M \geq 1$, in $m$ with deg $m=M$, in $\chi \bmod m$ of the third kind, and in $|t| \leq 1 / 2$,

$$
\left|f_{\chi}(t)\right| \gg M^{-7}(\log M)^{-3} \text {. }
$$

Proof. By (4.4), there exists $C>0$ such that

$$
\left|f_{\chi}(t)\right| \geq C M^{-1 / 4}(\log M)^{-3 / 4}|t-1 / 2|^{3 / 4} \text {. }
$$

Let $t_{0}, 0<t_{0}<1 / 2$, be the unique solution of

$$
M^{2}=\frac{3}{4} C M^{-1 / 4}(\log M)^{-3 / 4}\left|t-\frac{1}{2}\right|^{-1 / 4}: t_{0}=\frac{1}{2}-\left(\frac{3}{4}\right)^{4} C^{4} M^{-9}(\log M)^{-3} \text {. }
$$


Then

$$
\left|f_{\chi}(1 / 2)\right| \geq\left|f_{\chi}\left(t_{0}\right)\right|-M^{2}\left(1 / 2-t_{0}\right)
$$

from (4.5), and this is $\geq\left(\frac{3}{4}\right)^{3} \frac{1}{4} C^{4} M^{-7}(\log M)^{-3}$ from (4.4). Now for $\left|\frac{1}{2}-t\right|<\frac{1}{10}\left|\frac{1}{2}-t_{0}\right|$

$$
\left|f_{\chi}(t)\right| \geq\left|f_{\chi}\left(t_{0}\right)\right|-\frac{1}{10} M^{2}\left|\frac{1}{2}-t_{0}\right| \geq\left(\frac{3}{4}\right)^{3}\left(\frac{1}{4}-\frac{1}{10}\right) C^{4} M^{-7}(\log M)^{-3} .
$$

For $\left|t-\frac{1}{2}\right| \geq \frac{1}{10}\left|t_{0}-\frac{1}{2}\right|$, though,

$$
\begin{aligned}
\left|f_{\chi}(t)\right| & \geq C M^{-1 / 4}(\log M)^{-3 / 4}\left|t-\frac{1}{2}\right|^{3 / 4} \quad \text { by }(4.4), \\
& \geq\left(\frac{3}{4}\right)^{3}\left(\frac{1}{10}\right)^{3 / 4} C^{4} M^{-7}(\log M)^{-3} \text {. }
\end{aligned}
$$

Thus uniformly in $M, m, \chi \bmod m$ of the third kind, and for $t$, $|t|<1 / 2$,

$$
\left|f_{\chi}(t)\right| \geq C_{1} M^{-7}(\log M)^{-3} .
$$

The lemma follows by the continuity of the $f_{\chi}(t)$.

Now

$$
f_{\chi}(t)^{(n)}=\sum_{j=n}^{M-1} C_{J} \frac{n !}{j !} t^{J-n}
$$

so $\left|f_{\chi}(t)^{(n)}\right| \leq(2 M)^{n+1}$ in $|t| \leq 1 / 2$. Thus for $|v| \leq M^{-9}$ and $|T|=1 / 2$ we have

$$
\begin{aligned}
f_{\chi}(T+v) & =f_{\chi}(T)+O\left(\sum_{j=1}^{M-1} \frac{1}{j !}|v|^{j}(2 M)^{j+1}\right) \\
& \quad(\text { with the implicit constant }=1) \\
& =f_{\chi}(T)+O\left(M^{2}|v|\right) .
\end{aligned}
$$

Thus uniformly in $M, m$, and $\chi$,

$$
f_{\chi}(t) \neq 0 \quad \text { in }|t| \leq 1 / 2+C_{2}\left(M^{-9}(\log M)^{-3}\right)
$$

for some $C_{2}>0$.

5. Conclusions. We now expand (3.5) as a series in $t$, and estimate the coefficient of $t^{n-1}$.

From $\chi_{0}$, we get

$$
\sum_{n=1}^{\infty} 2^{n} t^{n-1}+\sum_{k=1}^{M} \frac{1}{k} I_{m}(k) \sum_{j=0}^{k-1} \sum_{n=1}^{\infty} t^{n-1} e\left(\frac{(n-1) j}{k}\right),
$$


so the coefficient of $t^{n-1}$ is

$$
2^{n}+\sum_{k=1}^{M} \frac{1}{k} I_{m}(k) \sum_{j=0}^{k-1} e\left(\frac{(n-1) j}{k}\right) .
$$

Now $\left|\sum_{j=0}^{k-1} e((n-1) j / k)\right| \leq k$, so the second term of (5.2) is $O\left(\sum_{k=1}^{M} I_{m}(k)\right)$. Now trivially this latter is $O(M)$. (A little thought shows it to be $O(M / \log M)$ but we have larger errors elsewhere.) Thus in (3.5) the coefficient of $t^{n-1}$ due to $\chi_{0}$ is

$$
2^{n}+O(M) \text {. }
$$

The expansion of the rest of (3.5) works out to $\sum_{n=1}^{\infty} r_{n} t^{n-1}$, where

$$
\begin{aligned}
r_{n} & =\sum_{\substack{\bmod _{\chi \neq \chi_{0}} \\
\chi(b)}} \frac{1}{w \in \Omega_{\chi}} \frac{-N(w)}{w}\left(\frac{1}{w}\right)^{n-1} \\
& =-\sum_{\substack{\chi \neq \chi_{0} m \\
\chi \neq \chi_{0}}} \frac{N(w)}{\chi(b)} w^{-n} .
\end{aligned}
$$

Now $|w| \geq 1 / 2+C_{2} M^{-9}(\log M)^{-3}$. Thus

$$
\left|r_{n}\right| \leq M \phi(m) 2^{n} \exp \left(-C_{3} n M^{-9}(\log M)^{-3}\right) .
$$

Now from (5.5), (5.3), and (3.8) we have

$$
\begin{aligned}
n \phi(m) & \left(I_{b}(n)+O\left(\frac{1}{n} 2^{n / 2}\right)\right) \\
= & 2^{n}+O(M)+O\left(M \phi(m) 2^{n} \exp \left(-C_{3} n M^{-9}(\log M)^{-3}\right)\right) .
\end{aligned}
$$

The theorem follows upon renumbering the constants.

\section{REFERENCES}

[1] L. Carlitz, A theorem of Dickson on irreducible polynomials, Proc. Am. Math. Soc., 3 (1952), 693-700.

[2] E. Landau, Elementary Number Theory, Chelsea, NY, 1966.

[3] Saburô Uchiyama, Sur les polynomes irréductibles dans un corps fini I, Proc. Japan Acad., 30 (1954), 523-527.

[4] _ , II, Proc. Japan Acad., 31 (1955), 267-269.

Received December 28, 1984. 



\section{PACIFIC JOURNAL OF MATHEMATICS EDITORS}

V. S. VARADARAJAN

(Managing Editor)

University of California

Los Angeles, CA 90024

HERBERT CLEMENS

University of Utah

Salt Lake City, UT 84112

R. FINN

Stanford University

Stanford, CA 94305
HERMANN FLASCHKA

University of Arizona

Tucson, AZ 85721

RAMESH A. GANGOLLI

University of Washington

Seattle, WA 98195

VAUGHAN F. R. JONES

University of California

Berkeley, CA 94720

ROBION KIRBY

University of California

Berkeley, CA 94720
C. C. MOORE

University of California

Berkeley, CA 94720

H. SAMELSON

Stanford University

Stanford, CA 94305

HAROLD STARK

University of California, San Diego

La Jolla, CA 92093

\section{ASSOCIATE EDITORS}

R. ARENS

E. F. BECKENBACH

B. H. NEUMANN (1906-1982)
F. WOLF

K. YOSHIDA

\section{SUPPORTING INSTITUTIONS}

UNIVERSITY OF ARIZONA

UNIVERSITY OF BRITISH COLUMBIA

CALIFORNIA INSTITUTE OF TECHNOLOGY

UNIVERSITY OF CALIFORNIA

MONTANA STATE UNIVERSITY

UNIVERSITY OF NEVADA, RENO

NEW MEXICO STATE UNIVERSITY

OREGON STATE UNIVERSITY
UNIVERSITY OF OREGON UNIVERSITY OF SOUTHERN CALIFORNIA

STANFORD UNIVERSITY

UNIVERSITY OF HAWAII

UNIVERSITY OF TOKYO

UNIVERSITY OF UTAH

WASHINGTON STATE UNIVERSITY

UNIVERSITY OF WASHINGTON 


\section{Pacific Journal of Mathematics}

\section{Vol. 123, No. $1 \quad$ March, 1986}

Maria Emilia Alonso García, A note on orderings on algebraic varieties $\ldots \ldots 1$

F. S. De Blasi and Józef Myjak, On continuous approximations for

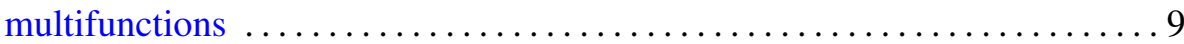

Frank Albert Farris, An intrinsic construction of Fefferman's CR metric . . . 33 Antonio Giambruno, P. Misso and Francisco César Polcino Milies, Derivations with invertible values in rings with involution $\ldots . \ldots . \ldots .47$

Dan Haran and Moshe Jarden, The absolute Galois group of a pseudo real

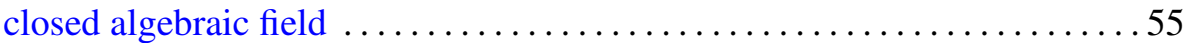

Telemachos E. Hatziafratis, Integral representation formulas on analytic

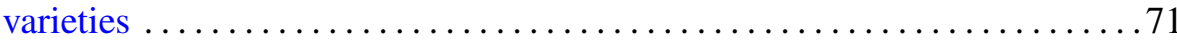

Douglas Austin Hensley, Dirichlet's theorem for the ring of polynomials

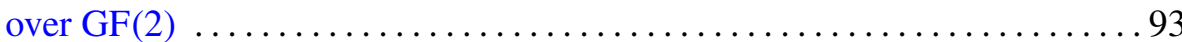

Sofia Kalpazidou, On a problem of Gauss-Kuzmin type for continued

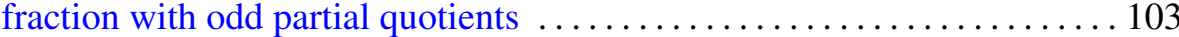

Harvey Bayard Keynes and Mahesh Nerurkar, Ergodicity in affine

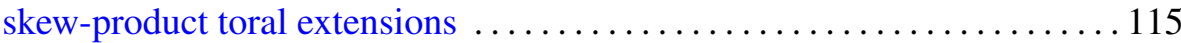

Thomas Landes, Normal structure and the sum-property $\ldots \ldots \ldots \ldots \ldots 127$

Anthony To-Ming Lau and Viktor Losert, Weak*-closed complemented invariant subspaces of $L_{\infty}(G)$ and amenable locally compact groups ...149 Andrew Lelek, Continua of constant distances in span theory . . . . . . . . 161 Dominikus Noll, Sums and products of $B_{r}$ spaces $\ldots \ldots \ldots \ldots \ldots \ldots \ldots \ldots$ Lucimar Nova, Fixed point theorems for some discontinuous operators 189

A. A. S. Perera and Donald Rayl Wilken, On extreme points and support points of the family of starlike functions of order $\alpha$

Massimo A. Picardello, Positive definite functions and $L^{p}$ convolution operators on amalgams ........................

Friedrich Roesler, Squarefree integers in nonlinear sequences ......... 223

Theodore Shifrin, The osculatory behavior of surfaces in $\mathbf{P}^{5}$ 